\title{
Pacific
}

Journal of

Mathematics

\section{AN EQUIVALENCE RELATION FOR CODIMENSION ONE FOLIATIONS OF 3-MANIFOLDS}

\author{
SANDRA SHIELDS
}

Volume $175 \quad$ No. 1 


\title{
AN EQUIVALENCE RELATION FOR CODIMENSION ONE FOLIATIONS OF 3-MANIFOLDS
}

\author{
SANDRA L. SHIELDS
}

\begin{abstract}
We consider codimension one foliations of closed, Reimannian 3-manifolds. We show all branched surfaces constructed from a foliation and a transverse flow are conjugate. We use branched surfaces to define an equivalence relation on foliations transverse to the same nonsingular flow. Under this relation, foliations in the same equivalence class need not be topologically conjugate yet they will share important qualitative properties.
\end{abstract}

\section{Introduction.}

The purpose of this paper is to define an equivalence relation for codimension one foliations of closed, orientable, Riemannian 3-manifolds. We use branched surfaces constructed from foliations with transverse flow $\phi$ to define an equivalence relation on foliations transverse to $\phi$. One of our conditions for two foliations to be equivalent is that both foliations may be used to construct the same branched surface. In [5], Penner gives a similar relation on laminations using Thurston's train tracks [10]. This, in part, motivated our definition. However, unlike Penner's relation, foliations in the same equivalence class need not be topologically conjugate.

If a branched surface $W$ is constructed from a foliation $f$ it can be shown that every foliation sufficiently close to $f$ ( in $C^{1}$ metric defined by Hirsch [4]) can be used to constuct $W$. However, because of additional conditions that we require for equivalence, arbitrarily close foliations may not be equivalent to $f$. These additional conditions are necessary to ensure that foliations in the same equivalence class share certain topological properties such as the existence of a compact leaf or the existence of a covering by a trivial product of hyperplanes. To illustrate the former, we consider a lower dimensional analog. All foliations of the torus, induced by lines in $R^{2}$ with fixed rational slope (by taking the quotient over the integer lattice) are equivalent under our definition. Yet they are not equivalent to any foliation induced by lines with arbitrary close irrational slope.

In Section I, we give a brief outline of the construction of a branched surface from a foliation using a transverse flow and a finite set of surfaces 
imbedded in the leaves. This outline is sufficient for understanding the results in this paper. However, a detailed description of this construction is given by Christy and Goodman in [1]. In Section II, we look at properties of branched surfaces constructed from foliations. In Section III, we extend Penner's moves on train tracks to moves on branched surfaces. We show that all branched surfaces constructed from a foliation using a minimal number of surfaces can be made diffeomorphic using these moves. This we use to show the relation defined in Section IV is an equivalence relation. We end with a brief look at the relationship between topologically conjugate foliations and equivalent foliations.

\section{The construction of a branched surface from a foliation.}

For this paper we will only consider codimension one foliations of closed, orientable, Riemannian 3-manifolds and their lower dimensional analogs (codimension one foliations of the torus). We may construct a branched surface from a foliation $f$ of a closed manifold $M$ using a continuous, transverse flow $\phi$. We shall use both $(f, \phi)$ and $(M, f)$ to denote this foliation, depending on the context. We choose a set, $\Delta=\left\{D_{i}\right\}_{i=1, \ldots n}$, of compact, planar surfaces with boundary that are imbedded in the leaves of $f$ and satisfy the following general position requirements with respect to $\phi$ :

I. The orbit $\phi(x, t)$ of every $x \in M$ meets the interior of some surface in $\Delta$ at some $t>0$.

II. For each $i$, the orbits of at most finitely many points in $\partial D_{i}$ meet the boundary of a surface in $\Delta$.

III. Any orbit meets at most two points that are in the boundary of a surface in $\Delta$.

It is worth noting that $I$ is equivalent to the requirement that the orbit of every point meets the interior of some surface of $\Delta$ at some $t<0$ since $M$ is compact and the limit set of any orbit is invariant under the flow.

We remove the interior of the surfaces in $\Delta$ to obtain a manifold $M^{*}$ imbedded in $M$. We open the components of $M-M^{*}$ slightly and take the closure of the resulting imbedded manifold. This gives a manifold $N(W)$ with boundary which has a foliation $f^{*}$ induced by $f$. The leaves of $f^{*}$ are tangent to the boundary of $N(W)$. We may construct $N(W)$ so its complement in $M$ is the union of lens-shaped 3-manifolds, $\left\{B_{i}\right\}_{i=1, \ldots n}$, and the upper and lower hemispheres in the boundary of each are identified to recover $(M, f)$ from $\left(N(W), f^{*}\right)$. See Figure 1.1. The foliation $f^{*}$ has a transverse flow $\phi^{*}$ induced by $\phi$. By the way we chose the elements of $\Delta$, all orbits of $\phi^{*}$ are homeomorphic to closed, connected subsets of $\mathfrak{R}$. These orbits are called the "fibers" of $N(W)$. 


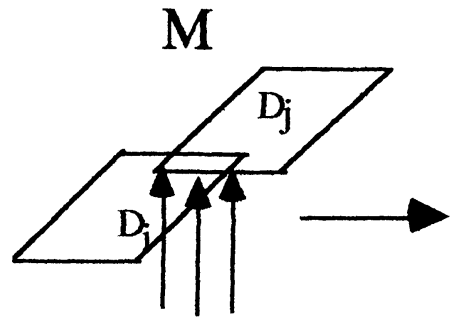

$\phi$

\section{$\mathbf{N}(\mathbf{W})$}

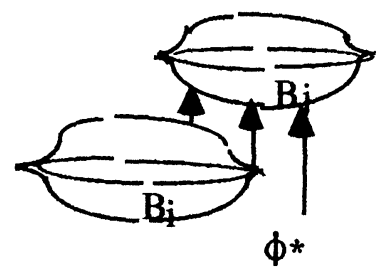

Figure 1.1.

We form the quotient space $N(W) / \sim$ where $x \sim y$ if and only if both $x$ and $y$ lie on the same orbit of $\phi^{*}$. By expanding the lens-shaped components of $M-N(W)$ so that each fiber of $N(W)$ is contracted to a point, we obtain an imbedding of the quotient space. This imbedding is the branched surface $W$. $W$ inherits a transverse orientation from the oriented fibers of $N(W)$.

It is worth noting that Christy and Goodman's technique, which was described above, may be generalized to construct a branched surface from any set of imbedded compact surfaces. However, for our purposes we assume that the compact surfaces in $\Delta$ are planar imbeddings.

Given a foliation with transverse flow, $(f, \phi)$, we let $n(f, \phi)$ be the minimal number of planar surfaces required to construct a branched surface from $(f, \phi)$. That is, $n(f, \phi)$ is the minimal number of surfaces necessary to satisfy the general position requirements. In Appendix A, we describe a straightforward procedure for calculating $n(f, \phi)$ from any set, $X=\left\{C_{i}\right\}_{i=1 \ldots n}$, of planar surfaces satisfying the general position requirements. (For example, if we have a covering of $(M, f)$ by foliation boxes, we may take $X$ to be any set that contains exactly one slice from each box, modified slightly to satisfy conditions II and III.) For this procedure we use $X$ to construct a branched surface $V$ from $(f, \phi)$ and then take successive modifications of $X$, that are determined by $V$. We do this until $X$ can no longer be modified in the prescribed manner. It will then have $n(f, \phi)$ elements. Since $V$ tends to become more complex for larger values of $n$, this procedure is more practicat when we can choose a relatively small $X$ to start.

We will henceforth consider only those branched surfaces that can be constructed from $n(f, \phi)$ planar surfaces using Christy and Goodman's technique. 


\section{Branched surfaces constructed from a foliation.}

The branched surfaces we obtain by this construction are the same as those in [3] and they are more restricted than those in [11]. Each branched surface $W$ is a transversely oriented 2-manifold except on a nonmanifold subset consisting of "branch points". This subset is called "branch set". The connected components of $W$-(branch set) are called the "sectors" of $W$.

The elements of $\Delta$ may be chosen large enough to ensure the branch set is connected and intersects itself transversely at least once. We will assume all branched surfaces have these properties.

By construction, each transversely oriented neighborhood of a point in $W$ is locally modeled on one of the following:
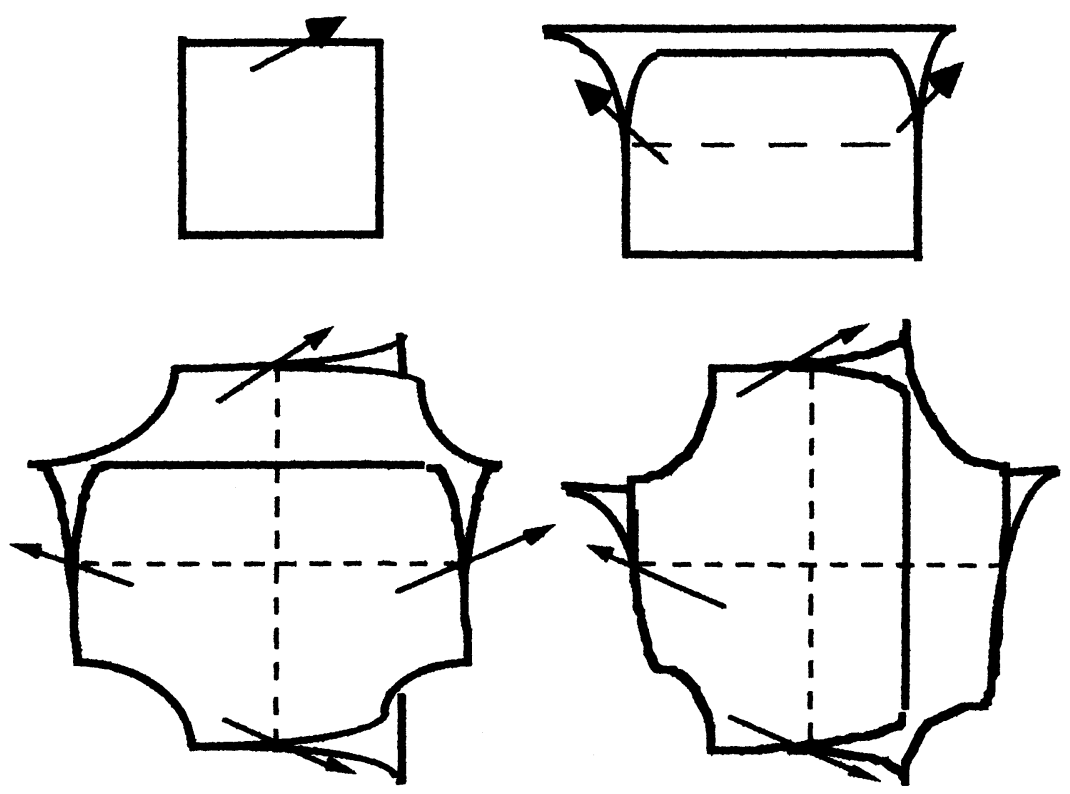

Figure 2.1.

Each local model projects horizontally onto a vertical model of $\mathfrak{R}^{2}$. Therefore, $T \mathfrak{R}^{2}$ induces a tangent bundle on $W$ when we pull back each local projection.

We may thicken any $x \in W$ along the transverse direction to recover the fiber of $N(W)$ that was contracted to $x$ in the construction of $W$. (We say each point of this fiber lies "over" $x$ ). We may reconstruct $N(W)$ by thickening $W$ in this manner. (See Figure 2.2.) 

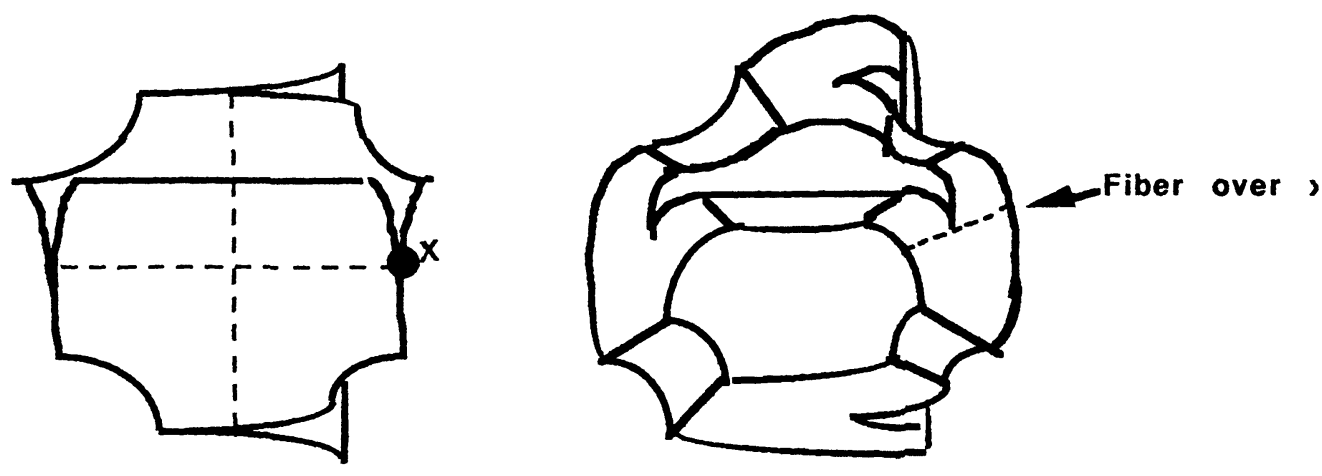

Figure 2.2. The diagram above shows how we thicken $W$ locally to reconstruct the "fiber" heighborhood, $N(W)$.

Formally, a curve in $W$ (or $N(W)$ ) is a map from a connected subset of $\mathfrak{R}$ into $W(N(W)$ respectively). However, we will consider it as the image of such a map, where the map gives the curve its parameterization. Similarily, an "integral curve" of a foliation is a map from a connected subset of $\mathfrak{R}$ into a leaf. However, for our purposes it will be the oriented image of such a map.

We only consider those foliations of $N(W)$ by surfaces (possibly branched) which are transverse to the fibers and tangent to $\partial N(W)$, such that $\{$ branch points of leaves $\}=\{$ branch points of $N(W)\}$. Figure 2.3 illustrates how such a foliation may appear locally.

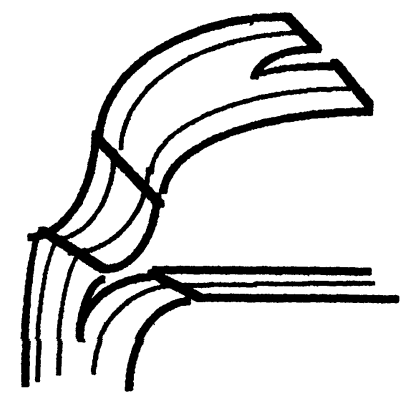

Figure 2.3.

If we have such a foliation, the boundary of each lens-shaped component of $M-N(W)$ is contained in a leaf. When we "collapse" the components 
of $M-N(W)$ by identifying the upper and lower hemispheres of the lensshaped 2-manifolds that bound them, a foliation of $N(W)$ yields a foliation $f^{\prime}$ of $M$. Further, the fibers induce a flow $\phi^{\prime}$ that is transverse to $f^{\prime}$. We say the foliation $\left(f^{\prime}, \phi^{\prime}\right)$ (or simply $f^{\prime}$ when the flow is clear) is "carried by $W^{\prime}$ ". In particular, if $W$ is constructed from $(f, \phi)$, then some foliation of $N(W)$ yields $f$ and we may collapse the complement of $N(W)$ in such a way that the fibers induce $\phi$. So in this case, $(f, \phi)$ is carried by $W$.

If $\left(f^{\prime}, \phi^{\prime}\right)$ is carried by $W, f^{\prime}$ may be used to construct $W$. The flow used for the construction is $\phi^{\prime}$. The surfaces used are the collapsed lens-shaped 2-manifolds that bound the complement of $N(W)$ in $M$.

Definition. Let $k^{*}$ be an immersion in $N(W)$ of a planar strip, $[0,1] \times$ $[-\epsilon, \epsilon]$. Suppose that for any lens-shaped component $B_{i}$ of $\overline{M-N(W)}$, $k^{*}(t, s) \cap \partial B_{i}=\phi$ whenever $t$ is sufficiently near (but not equal to) 0 or 1 . The image, $K=k^{*}([0,1] \times[-\epsilon, \epsilon])$, of the strip is a "connecting strip" of $N(W)$ if it is transverse to the fibers, and both ends, $k^{*}(\{0\} \times[-\epsilon, \epsilon])$ and $k^{*}(\{1\} \times[-\epsilon, \epsilon])$, are contained in the set of branch points of $N(W)$.

That is, connecting strip of $N(W)$ is simply an immersed strip, transverse to the fibers, with $\partial N(W)$ branching from both its ends. (See shaded region in Figure 2.4.)

Restrictions of different foliations of $N(W)$ to a local neighborhood containing a connecting strip may vary. For example, there are three canonical foliations of the local neighborhood in Figure 2.4. They are shown in Figure 2.5 .

It is worth noting that a foliation of a local neighborhood of $N(W)$ does not necessarily complete to a foliation of $N(W)$. In particular, if completion requires nontrivial holonomy along the boundary of an imbedded disk, then by Reeb stability [7] it is not possible.

Let $\pi_{W}: N(W) \rightarrow W$ be the continuous map induced by the quotient map from $N(W)$ onto $N(W) / \sim$ (i.e. $\pi_{W}$ is the map that collapses each fiber of $N(W)$ to a point in $W$ ). For every connecting strip $K_{\gamma}$, we associate curves $\gamma$ in $W$ that have $W$ branching out of both ends and lie in the image under $\pi_{W}$ of $K_{\gamma}$. Each we call a "connecting curve". For our purposes, we need only consider connecting curves that are contained in the branched set and the connecting strips that correspond to such curves. It is often the case that for some foliation $(M, f)$ carried by $W$ a connecting strip $K_{\gamma}$ is contained in a leaf of the corresponding foliation of $N(W)$. In this case, we say $K_{\gamma}$ and the associated connecting curve are "strong connecting in $f$ ". 


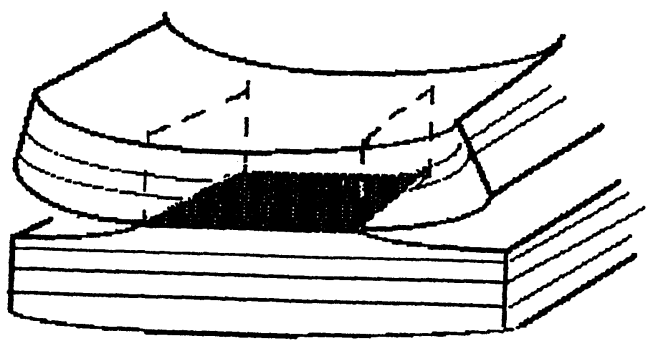

Figure 2.4. The shaded region in the figure is a "strong connecting strip" in the foliation shown.
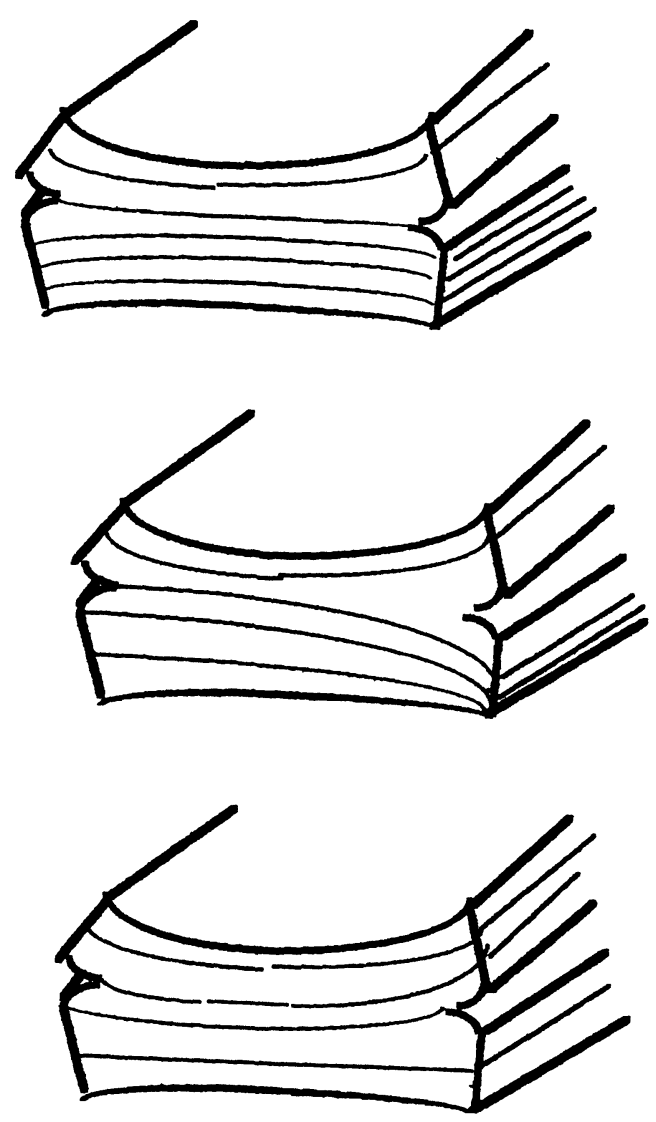

Figure 2.5. 
Definition. Now let $K_{\gamma}=k^{*}([0,1] \times[-\epsilon, \epsilon])$ be a connecting strip of $N(W)$ such that $k^{*}(\{0\} \times[-\epsilon, \epsilon]) \subseteq B_{i}$ and $k^{*}(\{1\} \times[-\epsilon, \epsilon]) \subseteq B_{j}$ for some lens-shaped components, $B_{i}$ and $B_{j}$ in $M-N(W)$.

Suppose $\alpha$ is a curve in a surface that is transverse to the fibers and suppose $\alpha$ is mapped by $\pi_{W}$ onto the corresponding connecting curve $\gamma$. Each curve containing $\alpha$ "crosses $K_{\gamma}$ in increasing (decreasing) order" if $\alpha$ extends from fibers through the lower (upper) hemisphere of $B_{i}$ and $\partial K_{\gamma}$, to a fiber through the upper (lower) hemisphere of $B_{j}$ and $\partial K_{\gamma}$ (where "above" and "below" are determined by the transverse orientation of $N(W)$ ). For the connecting curve $\gamma$ we say a curve in $W$ "crosses $\gamma$ in increasing (decreasing) order" if it properly contains $\gamma$ and its preimage under $\pi_{W}$ contains a curve that crosses $K_{\gamma}$ in increasing (decreasing) order. (See Figure 2.6.)
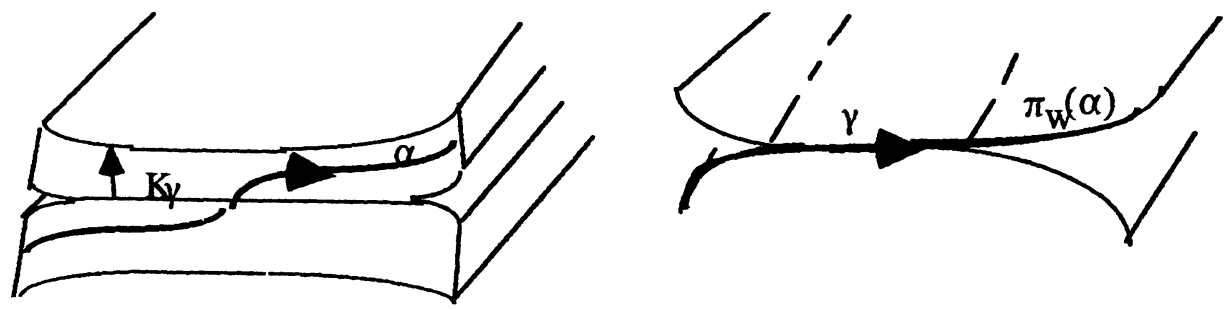

Figure 2.6. The figure at left shows a curve $\alpha$ crossing a connecting strip $K_{\gamma}$ in increasing order (orientation of fibers is indicated). The figure at right shows the projection of the curve. It is a curve crossing this corresponding connecting curve $\gamma$ in increasing order.

Definition. Let $f$ be a foliation carried by a branched surface $W$ and $f^{*}$ be the corresponding foliation of $N(W)$. A curve $\alpha$ on $W$ is an " $f$-curve" if it is the image under $\pi_{W}$ of an integral curve for $f^{*}$ (i.e., $\alpha$ is an $f$-curve if an integral curve of $f^{*}$ lies over $\alpha$ ). A surface $S$ (possibly branched) in $W$ is called an " $f$-surface" if it is the image under $\pi_{W}$ of a leaf in $f^{*}$ (i.e. $S$ is an $f$-surface if some leaf of $f^{*}$ lies over $S$ ).

If a connecting curve is strong connecting in $f$, then there is an $f$-curve that crosses it in increasing order and another that crosses it in decreasing order. Moreover, given an $f$-curve $\alpha$ and a second foliation $f^{\prime}$ carried by 'W if $\alpha$ is not an $f^{\prime}$-curve, then there exists a connecting curve in $W$ crossed by $\alpha$, that is not crossed any $f^{\prime}$-curve in the same order. This is straightforward to verify. We will use this observation to prove Lemma 4.1 .

Definition. Two branched surfaces, $W_{1}$ and $W_{2}$, imbedded in $M$ are 
"conjugate" if there is an orientation preserving diffeomorphism $g$ of $M$ that maps $W_{1}$ injectively onto $W_{2}$ and maps smooth submanifolds of $W_{1}$ onto smooth submanifolds of $W_{2}$. The map $g$ is called a "conjugacy map". Suppose $W_{1}$ carries $\left(f_{1}, \phi\right)$ and $W_{2}$ carries $\left(f_{2}, \phi\right)$. If $g$ maps the set of $f_{1}$-curves in $W_{1}$ injectively onto the set of $f_{2}$-curves in $W_{2}$, then we say $g$ "respects the $f_{1}-f_{2}$ structure" and the branched surfaces, $W_{1}$ and $W_{2}$, are " $f_{1}-f_{2}$ counterparts".

For simplicity, we will refer to conjugacy maps from $W_{1}$ onto $W_{2}$, with the underlying assumption that each extends to a diffeomorphism of $M$.

\section{Extending and contracting surfaces of $\Delta$.}

Let $W$ be a branched surface constructed from $(f, \phi)$ and let $f^{*}$ be the corresponding foliation of $N(W)$. In this section we discuss the effect on $W$ of extending or contracting a surface in $\Delta$. Suppose $D \in \Delta$ is contained in the leaf $L$. When we "extend" $D$, we replace $D$ with a compact, connected, planar surface in $L$ that contains $D$. When we "contract" $D$ we replace it with a compact, connected, planar surface contained in $D$. We require that these extensions and contractions yield a set that satisfies the general position requirements for the construction of a branched surface from $(f, \phi)$. After we extend or contract an element in $\Delta$, the branched surface we construct from $f$ (using this modified set) may be slightly different from $W$. We say $W$ has been modified by a "move in $f$ ". We note that the moves presented here are upper dimensional analogs of "zipping" and "unzipping" train tracks [5].

The effect of an extension on $N(W)$ is to enlarge some component of $M-N(W)$ by further splitting the leaf of $f^{*}$ containing it. For example, the upper diagram in Figure 3.1 depicts a local neighborhood in $N(W)$. When we split the indicated leaf further, we obtain the fiber neighborhood shown below it. This corresponds to splitting $W$ to enlarge a component of its complement. The upper diagram in Figure 3.2 shows a portion of the branched surface carrying the foliation $f$. It is the image under $\pi_{W}$ of the local neighborhood in Figure 3.1. The lower diagram in Figure 3.2 is the modified portion of the branched surface that we have after the extension. As another example, we split the local branched surface in Figure 3.3 along the indicated curve. This splitting also corresponds to an extension of a surface in $\Delta$. The effect of a contraction on $N(W)$ is to decrease the size of some component in $M-N(W)$ by pinching together subsets of its upper and lower hemisphere. This corresponds to pinching regions of $W$ together in such a way that we do not change the number of components in its complement. All splits by extensions in $f$ may be reversed by contracting the extended surface back to its initial state. 

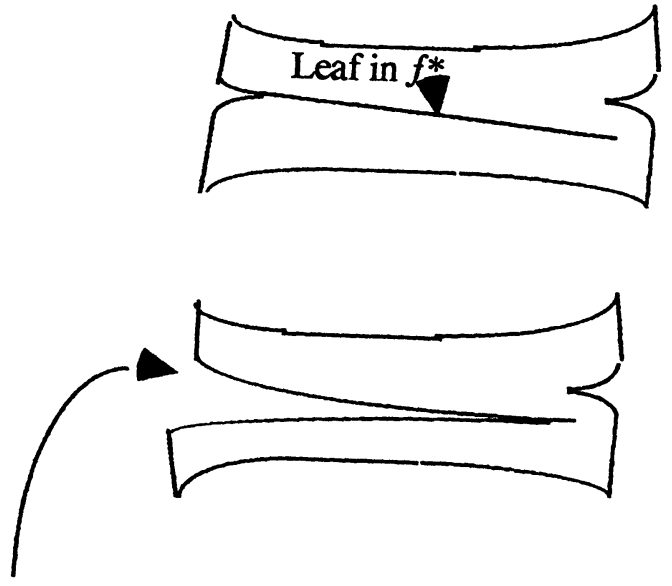

Figure 3.1. Effect of extending a surface in $\Delta$ along the corresponding leaf of $f$.
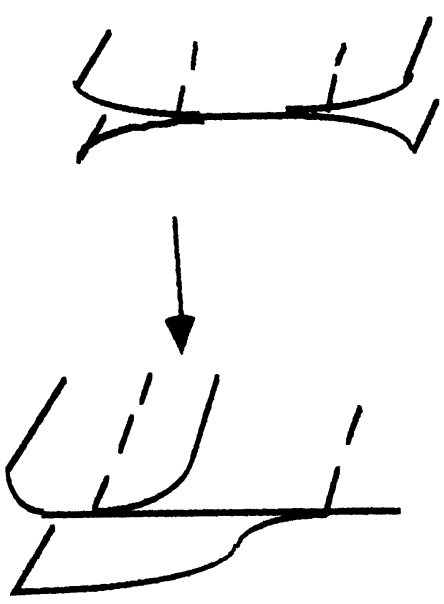

Figure 3.2.
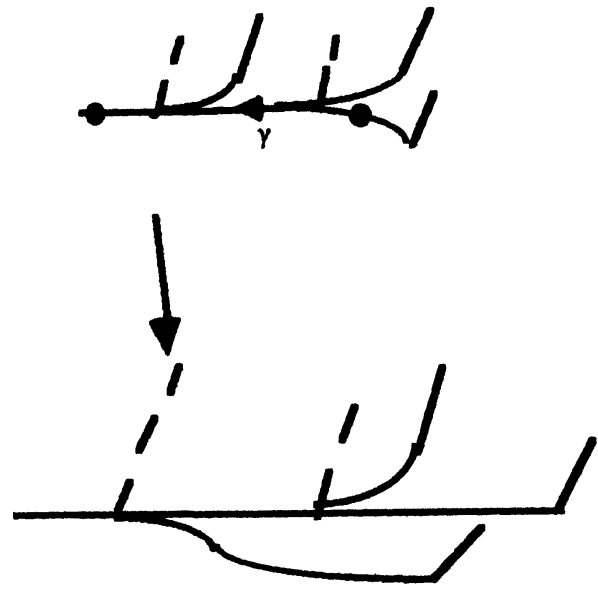

Figure 3.3.

If $\gamma$ in $W$ is a strong connecting curve in $f$, then we may enlarge some component in the complement of $N(W)$ by splitting $N(W)$ along the corresponding connecting strip. (This corresponds to extending some surface $D \in \Delta$.) See Figure 3.4. If $\gamma$ connects 2 distinct components of $M-W$, then this split may be reversed by deleting the appropriate (connected) subset in 
the interior of $D$. This pinches the branched surface back together along $\gamma$. In general, when we contract a surface in $\Delta$ to delete a connected subset of its interior, the branched surface is pinched together to create a strong connecting curve $f$.

effect on $N(W)$

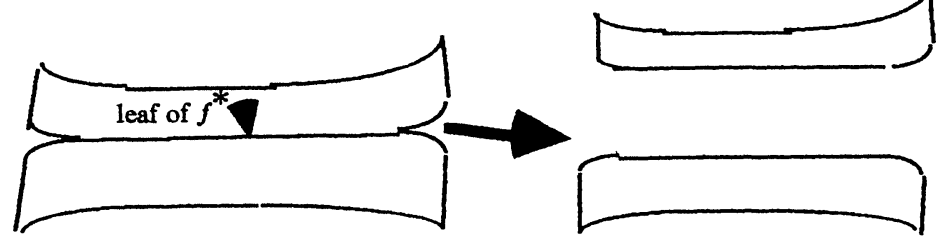

splitting of $W$
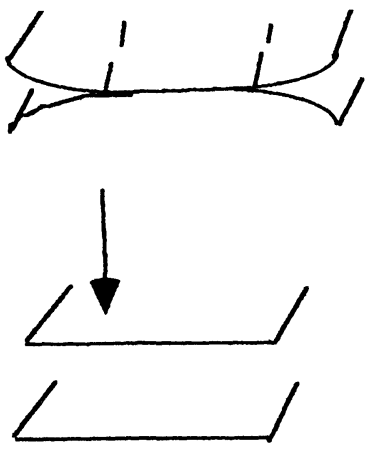

Figure 3.4.

Definition. Given surfaces, $D$ and $D^{\prime}$, transverse to the fibers in $N(W)$, we say " $D$ covers $D^{\prime}$ in $N(W)$ " if we may continuously project $D$ along the fibers, onto a subset of $N(W)$ that contains $D^{\prime}$.

The third type of modification of $W$ that we consider, a "substitution in $f^{\prime \prime}$, results when some $D \in \Delta$ is replaced by a surface $E$, where some extension of $E$ is covered by an extension of $D$ and contains a loop whose preimage under this covering is not closed.

Recall that we are only considering branched surfaces constructed from $(f, \phi)$ using $n(f, \phi)$ surfaces in leaves of $f$, where $n(f, \phi)$ is the minimal number of such surfaces necessary to satisfy the general position requirements.

Proposition 3.1. If two branched surfaces $W$ and $V$ are constructed from $(f, \phi)$, then they may be made conjugate by moves in $f$.

That is, we may modify $W$ and $V$ using moves in $f$ to obtain two conjugate branched surfaces.

Proof. Suppose $V$ and $W$ are constructed from $(f, \phi)$ using sets of surfaces, $X=\left\{C_{i}\right\}_{i=1 \ldots n}$ and $\Delta=\left\{D_{i}\right\}_{i=1 \ldots n}$, respectively, where $n=n(f, \phi)$. Consider the lens-shaped component of $M-N(W)$ that collapses to give $D_{i}$. The flow $\phi$ is transverse to $D_{i}$ and therefore induces a transverse orientation on the lens-shaped 2-manifold that bounds this component. Let $D_{i}^{+}\left(D_{i}^{-}\right)$ be the closure of the hemisphere which is "upper" ("lower") with respect 
to this orientation. (When we collapse this component of $M-N(W)$, each point $p^{+} \in D_{i}^{+}$is identified with a point $p^{-} \in D_{i}^{-}$.) Let $\Delta^{+}=\left\{D_{i}^{+}\right\}_{i=1 \ldots n}$. When we open $M$ along the interior of elements in $\Delta$ (the complement of $\left.M^{*}\right)$ and take the closure $N(W)$, the set $X$ becomes a set $X^{*}=\left\{C_{i}^{*}\right\}_{i=1 \ldots n}$ of surfaces in $N(W)$, transverse to the fibers and possibly branched.

Given $D_{j} \in \Delta$ we show that there exists $x \in \cup_{i=1 \ldots n}\left\{C_{i}\right\}$ such that its orbit $\phi(x, t)$ under $\phi$ meets $D_{j}$ before meeting the interior of some other element in $\Delta$ as $t \rightarrow-\infty$. We suppose this is not the case for some $j$. Every orbit (in particular, $\phi(x, t)$ for $x \in \cup_{i=1 \ldots n}\left\{\operatorname{int} C_{i}\right\}$ ) meets the interior of some element in $\Delta$ as $t \rightarrow-\infty$. Since the orbit of every point in $M$ must meet $\cup_{i=1 \ldots n}\left\{\operatorname{int} C_{i}\right\}$ at some $t>0$, every orbit meets the interior of some element of $\Delta-\left\{D_{j}\right\}$ as $t \rightarrow+\infty$. But then $\Delta-\left\{D_{j}\right\}$ also satisfies general position requirement $I$ for the construction of branched surface from $(f, \phi)$ yet has less than $n$ elements. We may extend the remaining elements slightly (if necessary) so $\Delta-\left\{D_{j}\right\}$ satisfies all 3 general position requirements. Since $\Delta-\left\{D_{j}\right\}$ has $n-1$ elements, we have a contradiction to our assumption that $n=n(f, \phi)$. So for every $j$, there exists an $x \in \cup_{i=1 \ldots n}\left\{C_{i}\right\}$ such that $\phi(x, t)$ meets $D_{j}$ before meeting the interior of some other element in $\Delta$ as $t \rightarrow-\infty$.

We may conclude that for every $D_{j}^{+} \in \Delta^{+}$there exists an $i$ such that some $x \in C_{i}^{*}$ projects along a fiber of $N(W)$ into $D_{j}^{+}$. We show that for any $D_{j}^{+}$ we may apply a move in $f$ so that some $C_{i}^{*} \in X^{*}$ covers $D_{j}^{+}$. This is trivial to show when $D_{j}$ intersects an element $C_{i}$ of $X$. In this case we simply extend $C_{i}$ to $D_{j} \cup C_{i}$. So we assume the elements of $\Delta$ do not intersect an element of $X$.

Given $D_{j}^{+}$, choose $i$ such that some $x \in C_{i}^{*}$ projects along a fiber of $N(W)$ into $D_{j}^{+}$. Suppose there is no extension of $C_{i}^{*}$ that covers $D_{j}^{+}$in $N(W)$. Then for each sufficiently large extension of $C_{i}^{*}$, the $f$-surface that contains $\pi_{W}\left(C_{i}^{*}\right)$ meets a branching of $W$ in $\pi_{W}\left(D_{j}^{+}\right)$and branches away from $\pi_{W}\left(D_{j}^{+}\right)$along $\pi_{W}\left(D_{k}^{+}\right)$, for some $k$. See Figure 3.5.

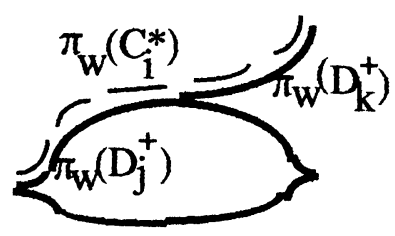

Figure 3.5.

Let $S$ be the sector of $W$ (i.e. connected component of $W$-\{branch set $\}$ ), 
that is adjacent to $\pi_{W}\left(C_{i}^{*}\right) \cap \pi_{W}\left(D_{j}^{+}\right)$and not met by this $f$-surface. Now, either: 1) there is some $C \in X$ that may be extended so that $\pi_{W}\left(C^{*}\right)$ intersects $\pi_{W}\left(C_{i}^{*}\right) \cap \pi_{W}\left(D_{j}^{+}\right)$and contains $S$ or 2) $\pi_{W}\left(C_{i}^{*}\right) \cap S=\emptyset$ for every $i$. In case $1, C^{*}$ is closer along fibers of $N(W)$ to $D_{j}^{+}$than $C_{i}^{*}$. Therefore, since the image under $\pi_{W}$ of an extension of $C^{*}$ intersects $\pi_{W}\left(C_{i}^{*}\right) \cap \pi_{W}\left(D_{j}^{+}\right)$, no extension of $C^{*}$ can couse $\pi_{W}\left(C_{i}^{*}\right)$ to branch away from $\pi_{W}\left(C_{i}^{*}\right) \cap \pi_{W}\left(D_{j}^{+}\right)$. We may extend $C$ so that the image of $C^{*}$ under $\pi_{W}$ covers $S$ and contains $\pi_{W}\left(C_{i}^{*}\right) \cap \pi_{W}\left(D_{j}^{+}\right)$. We then repeat the above argument using the modified $X$. In case 2 , we may contract $D_{j}$ so that the corresponding contraction of $D_{j}^{+}$does not intersect a fiber over $S$, and still have a set of surfaces which satisfies the first general position requirement with respect to $(f, \phi)$. (That is, we may modify $D_{j}$ to delete $S$, yet the orbit of any point in $\cup_{i=1 \ldots n}\left\{C_{i}\right\}$ and hence all points in $M$, will still meet the interior of some surface in $\Delta$ as $t \rightarrow-\infty$.) We then repeat the above argument using the modified $\Delta$. There are only a finite number of sectors of $W$. So, after a finite number of modifications of $\Delta$ and $X$, we obtain $C \in X$ which may be extended in $f$ so that $C^{*}$ covers the interior of $D_{j}^{+}$(possibly contracted) in $N(W)$.

Thus, we may modify $X$ using sufficiently large extensions, so that the interior of each element of $\Delta^{+}$(possibly contracted) in $N(W)$ is covered by some element of $X$. Now every element of $X$ covers the interior of some element of $\Delta^{+}$, since otherwise $X$ would contain more than $n(f, \phi)$ surfaces. We may further modify $X$ by contractions so that for every $i, C_{i}^{*}$ projects along fibers of $N(W)$ onto the interior of an element of $\Delta^{+}$yet not onto a planar subset that properly contains it. These modifications of $X$ may be constructed so it satisfies the general position requirements. After these modifications, we have a surjective map, $\tau:\left\{X^{*}\right\} \rightarrow\left\{\Delta^{+}\right\}$, where for any $C_{i}^{*} \in X^{*}, C_{i}^{*}$ covers the interior of $\tau\left(C_{i}^{*}\right)$ in $N(W)$. Now repeating the above argument using $\pi_{V}$, the modified $X$, and the corresponding set $X^{-}=\left\{C_{i}^{-}\right\}_{i=1 \ldots n}$ of lower hemispheres for elements in $M-N(V)$, we obtain (after further modifications) a surjective map $\tau^{\prime}\left\{\Delta^{*}\right\} \rightarrow\left\{X^{-}\right\}$, where $D_{i}^{*}$ covers the interior of $\tau^{\prime}\left(D_{i}^{*}\right)$ in $N(V)$. If this cover does not extend to a homeomorphism from $D_{i}^{*}$ onto $\tau^{\prime}\left(D_{i}^{*}\right)$, then one of these surfaces contains a loop that is not covered by a loop in the other, and we replace the latter using a substitution in $f$. After this last set of modifications of $\Delta$ and $X, C_{i}^{*}$ homeomorphically covers $\tau\left(C_{i}^{*}\right)$ in $N(W)$. For each $j, C_{i}^{*}>\tau\left(C_{i}^{*}\right) \in \Delta^{+}$(where $>$ is determined by the relative position along the oriented fibers). Let $\sigma_{W}: N(W) \rightarrow M$ be the map which identifies upper and lower hemishperesin the lens-shaped 2-manifolds that bound the components of $M-N(W)$. Then $\Delta=\cup_{i=1 \ldots n}\left\{\sigma_{W^{*}} \tau\left(C_{i}^{*}\right)\right\}$ and for every $i, \sigma_{W^{*}} \tau\left(C_{i}^{*}\right)$ is diffeomorphic to $C_{i}$ via translation along the orbits of $\phi$. Without loss of generality, we assume $\Delta$ is indexed so $\sigma_{W^{*}} \tau\left(C_{i}^{*}\right)=D_{i}$ for all $i$. Then there is a diffeomor- 
phism of $M$ that translates $D_{i} \in \Delta$, along orbits of $\phi$, onto $C_{i} \in X$ for each $i$. Further, these orbits of points in $D_{i} \in \Delta$ meet $C_{i} \in X$ before meeting any other element of $X$. Since the construction of a branched surface depends only on the surfaces chosen and their relative position with respect to the flow, this diffeomorphism induces a conjugacy map from $W$ onto $V$.

\section{An equivalence relation.}

In this section we define a relation on foliations with the same transverse flow and show that this is an equivalence relation. For a foliation $f$ carried by a branched surface $W$, we shall let $f^{*}$ denote the foliation on $N(W)$ that yields $f$ when we collapse the complement of $N(W)$ in $M$. Given a set $\Delta=\left\{D_{i}\right\}_{i=1 \ldots n}$ used to construct a branched surface from $(f, \phi)$, we shall say $x \in M$ flows into $D_{i}$ under $\phi$ if as $t \rightarrow+\infty$, the orbit $\phi(x, t)$ meets int $D_{i}$ before meeting the interior of some other element of $\Delta$.

Recall that we only consider branched surfaces constructed from $(f, \phi)$ using $n(f, \phi)$ imbedded, compact, planar surfaces with boundary. Suppose $V$ and $W$ are constructed from $(f, \phi)$. By proposition 3.1, we may obtain conjugate branched surfaces, $V^{\prime}$ and $W^{\prime}$, from $V$ and $W$, respectively, by moves in $f$. We let $f_{W^{\prime}}^{*}$ be the foliation of $N\left(W^{\prime}\right)$ that yields $f$ when we collapse the complement of $N\left(W^{\prime}\right)$ in $M$. Similarily, we let $f_{V^{\prime}}^{*}$ be the foliation of $N\left(V^{\prime}\right)$ that yields $f$ when we collapse the complement of $N\left(V^{\prime}\right)$ in $M$. We assume $X^{\prime}=\left\{C_{i}\right\}_{i=1, \ldots n}$ is the set of (modified) surfaces used to construct $V^{\prime}$ and $\Delta^{\prime}=\left\{D_{i}^{\prime}\right\}_{i=1 \ldots n}$ is the set of (modified) surfaces used to construct $W^{\prime}$. It follows from the proof of Proposition 3.1 that we may assume each element of $\Delta^{\prime}=\left\{D_{i}^{\prime}\right\}_{i=1 \ldots n}$ flows injectively along orbits of $\phi$, onto an element of $X^{\prime}=\left\{C_{i}^{\prime}\right\}_{i=1 \ldots n}$. We will henceforth assume $X^{\prime}$ has been indexed so $D_{i}^{\prime}$ flows injectively onto $C_{i}^{\prime}$ for every $i$. For each $i$, let $A_{i}^{\prime}$ be the lens-shaped component in $M-N\left(V^{\prime}\right)$ that collapses to $C_{i}^{\prime}$ and $B_{i}^{\prime}$ be the lens-shaped component in $M-N\left(W^{\prime}\right)$ that collapses to $D_{i}^{\prime}$. It also follows from our proof of Proposition 3.1, that there exists a conjugacy map, $g: W^{\prime} \rightarrow V^{\prime}$, such that $g * \pi_{W^{\prime}}\left(\partial B_{i}^{\prime}\right)=\pi_{V^{\prime}}\left(\partial A_{i}^{\prime}\right)$. We have the following:

Lemma 4.1. If $g$ does not respect the $f-f$ structure, we may construct branched surfaces that are $f-f$ counterparts by replacing $C_{i}^{\prime} \in X^{\prime}$ and $D_{i}^{\prime} \in \Delta^{\prime}$ with some surface $E_{i}^{\prime}$ for selected values of $1 \leq i \leq n$.

Proof. Suppose the conjugacy map $g$ from $W^{\prime}$ onto $V^{\prime}$ does not map the set of $f$-curves of $W^{\prime}$ injectively onto the set of $f$-curves of $V^{\prime}$. Then, as we observed in Section II, $W^{\prime}$ has a connecting curve $\gamma$ such that either its image $g(\gamma)$ is crossed by $f$-curves in some order that $\gamma$ is not, or the converse holds. (E.g. $\gamma$ and $g(\gamma)$ are not both strong connecting in $f$.) Without loss 
of generality, we assume the former. Let $K_{\gamma}$ be a connecting strip in $N\left(W^{\prime}\right)$ corresponding to $\gamma$ and $K_{g(\gamma)}$ be the connecting strip in $N\left(V^{\prime}\right)$ corresponding $g(\gamma)$. Assume the branching at the two ends of $K_{\gamma}$ are contained in $\partial B_{i}^{\prime}$ and $\partial B_{j}^{\prime}$ respectively.

We show that $i=j$. If $i \neq j$, then neither $\gamma$ nor $g(\gamma)$ are strong connecting in $f$ since $W^{\prime}$ and $V^{\prime}$ are constructed from $n(f, \phi)$ surfaces. (Otherwise we could extend one of these surfaces so that the branched surface is split along the strong connecting strip. This would decrease the number of components in the complement of the branched surface. This is not possible since each surface used for the construction of a branched surface is obtained by collapsing one of these components.) Without loss of generality, suppose some $f$-curve in $W^{\prime}$ crosses $\gamma$ in increasing order. (If not, reverse the orientation of $\gamma$.) Then some integral curve of $f_{W^{\prime}}^{*}$ beginning in $\partial B_{i}^{\prime}$ crosses $K_{\gamma}$ in increasing order. Further, some $f$-curve of $V^{\prime}$ crosses $g(\gamma)$ in decreasing order. So, some integral curve of $f_{V^{\prime}}^{*}$ beginning in $\partial A_{i}^{\prime}$ crosses $K_{g(\gamma)}$ in decreasing order. Let $\sigma_{W^{\prime}}\left(K_{\gamma}\right)$ be the image of $K_{\gamma}$ when we collapse the complement of $N\left(W^{\prime}\right)$ in $M$ and let $\sigma_{V^{\prime}}\left(K_{g(\gamma)}\right)$ be the image of $K_{g(\gamma)}$ when we collapse the complement of $N\left(V^{\prime}\right)$ in $M$. Recall that $g$ is induced by a diffeomorphism of $M$ that maps each $\sigma_{W^{\prime}}\left(\partial B_{k}^{\prime}\right)=D_{k}^{\prime} \in \Delta^{\prime}$ onto $\sigma_{V^{\prime}}\left(\partial A_{k}^{\prime}\right)=C_{k}^{\prime} \in X^{\prime}$ by translation along orbits of $\phi$. Thus $K_{\gamma}$ and $K_{g(\gamma)}$ may be chosen so $\sigma_{W^{\prime}}\left(K_{\gamma}\right)$ flows injectively onto $\sigma_{V^{\prime}}\left(K_{g(\gamma)}\right)$. We may extend $C_{i}^{\prime}$ in $f$ so that $C_{i}^{\prime}$ flows injectively under $\phi^{-1}$, onto $\sigma_{W^{\prime}}\left(K_{\gamma}\right) \cup D_{i}^{\prime} \cup D_{j}^{\prime}$. See Figure 4.1. Now $D_{i}^{\prime}$ and $D_{j}^{\prime}$ in $\Delta^{\prime}$ can be replaced with this extended $C_{i}^{\prime}$ to obtain a modification of $\Delta^{\prime}$ that also satisfies the general position requirements for the construction of a branched surface from $(f, \phi)$. However, this modification of $\Delta^{\prime}$ has less elements than the original $\Delta^{\prime}$ used to construct $W^{\prime}$. This contradicts the fact that $n(f, \phi)$ surfaces were used to construct $W^{\prime}$. So, $i=j$.

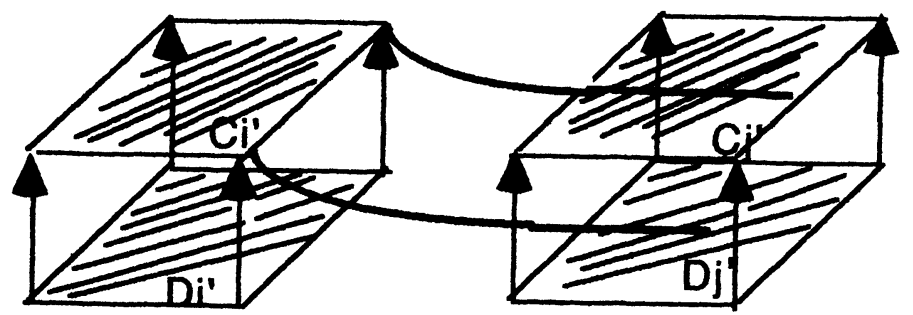

Figure 4.1. The directed segments are orbits of $\phi$. The bold lines indicate the extension of $C_{i}^{\prime}$ in $f$.

Without loss of generality, we again assume that $\gamma$ is crossed by $f$-curves 
in increasing order and $g(\gamma)$ is crossed by $f$-curves in decreasing order. As above, we may choose $K_{\gamma}$ and $K_{g(\gamma)}$ so that $\sigma_{W^{\prime}}\left(K_{\gamma}\right)$ flows injectively onto $\sigma_{V^{\prime}}\left(K_{g(\gamma)}\right)$. Let $\alpha(t)_{1 \leq t \leq 2 \pi}$ be an oriented immersion of $S^{1}$ in $M$ that is transverse to orbits of $\phi$ between $D_{i}^{\prime} \cup \sigma_{W^{\prime}}\left(K_{\gamma}\right)$ and $C_{i}^{\prime} \cup \sigma_{V^{\prime}}\left(K_{g(\gamma)}\right)$. So, for each $x \in \alpha, \phi(x, t)$ meets $C_{i}^{\prime} \cup \sigma_{V^{\prime}}\left(K_{g(\gamma)}\right)$ at some $t \geq 0$. If $k^{*}$ is a curve in $K_{g(\gamma)}$ such that $\pi_{V^{\prime}}\left[k^{*}(t)\right]=g(\gamma(t))$ for some parameterization of $k^{*}$ and $g(\gamma)$, we may choose $\alpha$ so that a connected subset of $\alpha$ flows into $\sigma_{V^{\prime}}\left(k^{*}\right)$. Let $I$ be part of an orbit of $\phi$, that extends from $\partial D_{i}^{\prime} \cap \sigma_{W^{\prime}}\left(K_{\gamma}\right)$ to $\partial C_{i}^{\prime} \cap \sigma_{V^{\prime}}\left(K_{g(\gamma)}\right)$ and intersects $\alpha$ at say $\alpha(0)=\alpha(2 \pi)$. See Figure 4.2. The holonomy map of each point on $I$ along $\alpha$ (i.e. along an integral curve of $f$ that transversely projects onto $\alpha$ ) has its image in $I$. Therefore, the holonomy map of $I$ along $\alpha$ has a fixed point. Since the leaves of $f$ are transverse to $\phi$, the leaf $L$ of $f$ through this point contains a surface $E_{i}^{\prime}$ that flows injectively onto $C_{i}^{\prime}$ under $\phi$ and injectively onto $D_{i}^{\prime}$ under $\phi^{-1}$. Therefore, we may use $E_{i}^{\prime}$ in place of $C_{i}^{\prime} \in X^{\prime}$ for the construction of $V^{\prime}$ and $E_{i}^{\prime}$ in place of $D_{i}^{\prime} \in \Delta^{\prime}$ for the construction of $W^{\prime}$. The branched surfaces we construct are conjugate to the original $W^{\prime}$ and $V^{\prime}$. The images under the respective conjugacy maps of both $\gamma$ and $g(\gamma)$ are strong connecting curves in $f$. Moreover, $A_{i}^{\prime}$ in $M-N\left(V^{\prime}\right)$ and $B_{i}^{\prime}$ in $M-N\left(W^{\prime}\right)$ now collapse to $E_{i}^{\prime}$. So any connecting curve with an end in $\pi_{V^{\prime}}\left(\partial A_{i}^{\prime}\right)$ is crossed by $f$-curves in the same order as a connecting curve with an end in $\pi_{W^{\prime}}\left(\partial B_{i}^{\prime}\right)$.

After substituting some $E_{i}^{\prime}$ as above for all necessary $1 \leq i \leq n$, the new $V^{\prime}$ and $W^{\prime}$ we construct are $f-f$ conjugate.

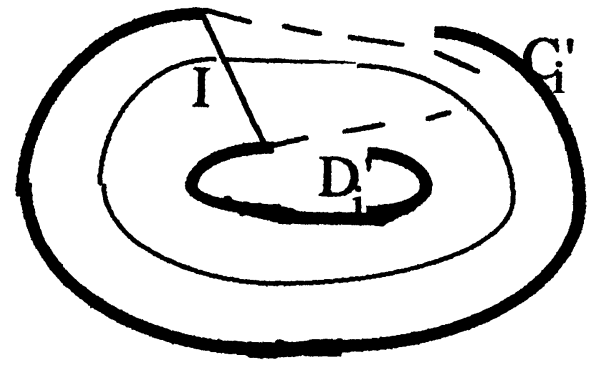

Figure 4.2. The bold lines indicate surfaces, $C_{i}^{\prime}$ and $D_{i}^{\prime}$, used to construct the branched surfaces. The dotted lines depict portions of the leaves that contain them.

When we replace $D_{i}^{\prime} \in \Delta^{\prime}$ (or $C_{i}^{\prime} \in X^{\prime}$ ) with $E_{i}^{\prime}$ as above, the branched surface we construct is conjugate to the original. We may think of this as 
changing a connecting curve in the original that is not strong connecting in $f$ to one that is strong connecting in $f$ (that is, it's image under the conjugacy map) by "altering" $\Delta^{\prime}$ ( $X^{\prime}$ respectively). If a connecting curve $\gamma$ can be changed to strong connecting by altering $\Delta^{\prime}$ ( $x^{\prime}$ respectively) we say it is "critically connecting in $f$ ". We will always assume that $E_{i}^{\prime}$ is chosen to ensure that the maximum number of critically connecting curves in $f$ are changed to strong connecting in $f$ when we alter $\Delta^{\prime}\left(X^{\prime}\right.$ respectively) with this substitution.

Let $W_{1}$ be a branched surface constructed from $\left(f_{1}, \phi\right)$ using $\Delta_{1}=$ $\left\{D_{i}^{1}\right\}_{i=1 \ldots n}$. Let $W_{2}$ be a branched surface constructed from $\left(f_{2}, \phi\right)$ using $\Delta_{2}=\left\{D_{i}^{2}\right\}_{\imath=1 \ldots n}$ such that $W_{1}$ and $W_{2}$ are $f_{1}-f_{2}$ counterparts. For $j \in\{1,2\}$, we represent its complement, $|j-2|+1$, by $j_{c}$. Suppose we modify $W_{j}$ using a sequence of substitutions in $f_{2}$. If each successive substitution in $\Delta_{j}$ can be chosen so that by altering $\Delta_{j_{c}}$ with similar substitutions, we obtain branched surfaces that are still $f_{1}-f_{2}$ counterparts, then we say $\left(f_{1}, W_{1}\right)$ and $\left(f_{2}, W_{2}\right)$ are "compatible". For example, if $\left(f_{1}, W_{1}\right)$ and $\left(f_{2}, W_{2}\right)$ are compatible then whenever we change connecting curves in $W_{j}$ that are not strong connecting (in $f_{j}$ ) to ones that are (by altering $\Delta_{j}$ ), we may subsequently do the same for the corresponding connecting curves in $W_{j_{c}}$ (by altering $\Delta_{j_{c}}$ ), without destroying the structure preserving property of conjugacy map. If all elements in $\Delta_{j}$ can be chosen in compact leaves of $f_{j}$, it follows from results in $[8]$ that $\left(f_{1}, W_{1}\right)$ and $\left(f_{2}, W_{2}\right)$ are compatible.

Definition. Given two foliations, $f_{1}$ and $f_{2}$, that are transverse to $\phi$, $\left(f_{1}, \phi\right) \sim\left(f_{2}, \phi\right)$ if the following conditions are satisfied:

A. $n\left(f_{1}, \phi\right)=n\left(f_{2}, \phi\right)$.

B. There exists a branched surface $W_{1}$ carrying $\left(f_{1}, \phi\right)$, that is an $f_{1}-f_{2}$ counterpart to a branched surface $W_{2}$ carrying $\left(f_{2}, \phi\right)$.

C. $W_{1}$ and $W_{2}$ may be chosen so that $\left(f_{1}, W_{1}\right)$ and $\left(f_{2}, W_{2}\right)$ are compatible. Condition $B$ in the definition of $\sim$ is sufficient to guarantee equivalent foliations share certain topological properties such as the existence of a compact leaf of a particular genus and the existence of a covering by a trivial product of hyperplanes in $\mathfrak{R}^{3}$ (Details can be found in [8] and [9].) We emphasize that compatibility is only required to ensure transitivity of conditions $\mathrm{A}$ and $\mathrm{B}$. We do not have an example that illustrates the necessity of condition $\mathrm{C}$ for transitivity. However, it seems likely that such examples exist.

Although compatability may be difficult to verify, it is often the case that there are substitutions in $\Delta_{j}$ which change all critically connecting curves in $f_{j}$ to strong connecting in $f_{j}$. In appendix $\mathrm{B}$ we discuss conditions under which it is sufficient for transitivity to check that alterations of this type do not destroy the structure preserving property of the conjugacy map. 
Theorem 4.2. $\sim$ is an equivalence relation.

Proof. It is obvious that $\sim$ is reflexive. The symmetry follows directly from the definitions.

The transitivity of condition $\mathrm{A}$ is immediate. To show transitivity of condition $\mathrm{B}$, we assume $W_{1}$ and $W_{2}$ are $f_{1}-f_{2}$ counterparts and $\left(f_{1}, W_{1}\right)$ and $\left(f_{2}, W_{2}\right)$ are compatible. Further, we assume $V_{2}$ and $V_{3}$ are $f_{2}-f_{3}$ counterparts and $\left(f_{2}, V_{2}\right)$ and $\left(f_{3}, V_{3}\right)$ are compatible. Suppose $W_{2}$ is constructed from $\left(f_{2}, \phi\right)$ using $\Delta=\left\{D_{i}\right\}_{i=1 \ldots n}$, and $V_{2}$ is constructed from $\left(f_{2}, \phi\right)$ using $X=\left\{C_{i}\right\}_{i=1 \ldots n}$. Since $W_{2}$ and $V_{2}$ are both constructed from $f_{2}$, they can be made conjugate by moves in $f_{2}$. So there exist conjugate branched surfaces, $W_{2}^{\prime}$ and $V_{2}^{\prime}$, obtained from $W_{2}$ and $V_{2}$, respectively, by moves in $f_{2}$. Suppose $W_{2}^{\prime}$ is constructed from $\left(f_{2}, \phi\right)$ using the modification, $\Delta^{\prime}=\left\{D_{i}^{\prime}\right\}_{i=1 \ldots n}$, of $\Delta$ and $V_{2}^{\prime}$ is constructed from $\left(f_{2}, \phi\right)$ using the modification, $X^{\prime}=\left\{C_{i}^{\prime}\right\}_{i=1 \ldots n}$, of $X$. By Lemma 4.1, if $W_{2}^{\prime}$ and $V_{2}^{\prime}$ are not $f_{2}-f_{2}$ counterparts, then for some $i$ we may change critically connecting curves of both $W_{2}^{\prime}$ and $V_{2}^{\prime}$ to strong connecting curves (in $f_{2}$ ) by replacing $D_{i}^{\prime} \in \Delta^{\prime}$ and $C_{i}^{\prime} \in X^{\prime}$ with a particular surface $E_{i}^{\prime}$. (It is worth noting that in this case $D_{i}^{\prime}+C_{i}^{\prime}$ are distinct so neither came from a substitution in $f_{2}$.) This surface $E_{i}^{\prime}$ flows injectively onto $D_{i}^{\prime}$ and $C_{i}^{\prime}$ under $\phi^{-1}$ and $\phi$, respectively. So $E_{i}^{\prime}$ can be modified by moves in $f_{2}$ so that it flows injectively onto $D_{i}$ under $\phi^{-1}$. We construct a new $W_{2}$ by substituting this modified $E_{i}^{\prime}$ for $D_{i} \in \Delta$. We may also modify $E_{i}^{\prime}$ so it flows injectively onto $C_{i}$ under $\phi$. We construct a new $V_{2}$ by substituting this modified $E_{i}^{\prime}$ for $C_{i} \in X$. Since $\left(f_{2}, W_{2}\right)$ and $\left(f_{1}, W_{1}\right)$ were originally chosen to be compatible, we may choose a new $W_{1}$ that is an $f_{1}-f_{2}$ counterpart to the new $W_{2}$. Similarily, since $\left(f_{2}, V_{2}\right)$ and $\left(f_{3}, V_{3}\right)$ were originally chosen to be compatible, we may choose a new $V_{3}$ that is an $f_{2}-f_{3}$ counterpart to the new $V_{2}$. By Lemma 4.1, at most $n$ new choices for $W_{2}+V_{2}$ are necessary to ensure the conjugate modifications $W_{2}^{\prime}$ and $V_{2}^{\prime}$ of $W_{2}$ and $V_{2}$ are $f_{2}-f_{2}$ counterparts. If we modify $W_{1}$ and $V_{3}$ as above at each stage, the moves in $f_{2}$ to obtain $W_{2}^{\prime}$ from the new $W_{2}$ correspond to the moves in $f_{1}$ on the new $W_{1}$. These moves in $f_{1}$ give a branched surface that is an $f_{1}-f_{2}$ counterpart to $W_{2}^{\prime}$. The moves in $f_{2}$ to obtain $V_{2}^{\prime}$ from the new $V_{2}$ correspond to moves in $f_{3}$ on the new $V_{3}$. These moves in $f_{3}$ give a branched surface that is an $f_{2}-f_{3}$ counterpart to $V_{2}^{\prime}$. It follows that some branched surface that carries $f_{1}$ (and is obtained from the new $W_{1}$ using moves in $f_{1}$ ), is an $f_{1}-f_{3}$ counterpart to some branched surface that carries $f_{3}$ (and is obtained from the new $V_{3}$ using moves in $f_{3}$ ).

Now, for the new choices of $W_{1}, W_{2}, V_{2}$ and $V_{3},\left(f_{2}, W_{2}\right)$ and $\left(f_{1}, W_{1}\right)$ are still compatible and $\left(f_{2}, V_{2}\right)$ and $\left(f_{3}, V_{3}\right)$ are still compatible. So, the transitivity of condition $\mathrm{C}$ follows from the transitivity of compatibility and 
the fact that compatibility is not affected by moves in the respective foliations.

For example, consider foliations, $f_{\alpha}$, of the torus induced by lines in $\mathfrak{R}^{2}$ with fixed rational slope $\alpha$ (when we take the quotient over integer lattice). For every $\alpha \neq 0$ such that $f_{\alpha}$ is transverse to a flow $\phi$ with fixed slope, $n\left(f_{\alpha}, \phi\right)=1$ (i.e., $\Delta_{\alpha}=\left\{D_{1}\right\}$ ). Moreover, the surface used for the construction of a branched surface from $\left(f_{\alpha}, \phi\right)$ can be chosen in any leaf. Since all leaves are compact, each pair of branched surfaces, $W_{\alpha_{1}}$ and $W_{\alpha_{2}}$, constructed from $\left(f_{\alpha_{1}}, \phi\right)$ and $\left(f_{\alpha_{2}}, \phi\right)$ respectively, can be made $f_{\alpha_{1}}-f_{\alpha_{2}}$ counterparts simply by extending in $f_{\alpha_{1}}$ and $f_{\alpha_{2}}$ when $\alpha_{1}$ and $\alpha_{2}$ are sufficiently close (no substitutions in $\Delta_{\alpha}$ are necessary). It is immediate that $\left(f_{\alpha_{1}}, W_{\alpha_{1}}\right)$ and $\left(f_{\alpha_{2}}, W_{\alpha_{2}}\right)$ are compatible. So when $\alpha_{1}$ and $\alpha_{2}$ are both rational and sufficiently close, $f_{\alpha_{1}}$ is equivalent to $f_{\alpha_{2}}$. Further, no foliation with fixed rational slope is equivalent to a foliation with fixed irrational slope.

As another example, consider the Reeb foliation $f_{R}$ of $S^{3}$ with a single toral leaf $C$. Let $\phi$ be a transverse Morse-Smale flow with two periodic orbits, one attracting and the other repelling, contained in different components of $S^{3}-C$. It is easy to verify that $n\left(f_{R}, \phi\right)=2$. Figure 4.3 shows a branched surface constructed from $\left(f_{R}, \phi\right)$ using two imbedded disks, one transverse to each periodic orbit. It can be shown that all foliations carried by this branched surface are equivalent to the Reeb foliation. It was shown in [8] that each of these foliations has a compact toral leaf.

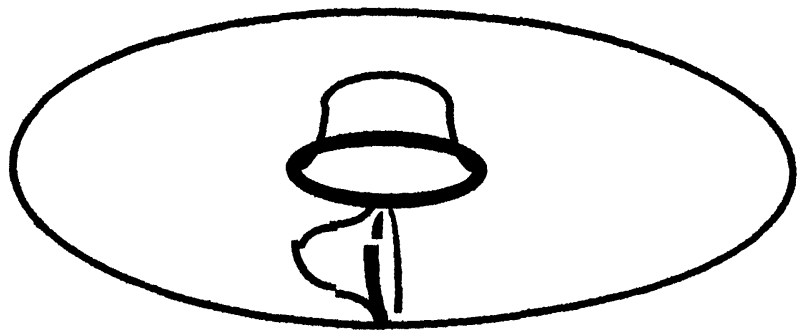

Figure 4.3.

We note that the relation, $\sim$, is different from topological conjugacy. As the above example suggests, there are many foliations that are equivalent under $\sim$ yet not topologically conjugate. In addition, we do not know whether $(f, \phi) \sim\left(f^{\prime}, \phi\right)$ whenever $f$ and $f^{\prime}$ are transverse to $\phi$ and topologically conjugate. 


\section{Appendix A.}

Here we use the techique described in Section III together with the proof of Proposition 3.1, to develop a procedure for calculating $n(f, \phi)$. We shall consider sets used to construct branched surfaces from $(f, \phi)$. When we say a point $x \in M$ flows into an element of one such set under $\phi$, we shall mean that the orbit $\phi(x, t)$ meets the interior of this element as $t \rightarrow+\infty$ before meeting the interior of any other element of the set.

We begin with any branched surface $V$ constructed from $(f, \phi)$ using a set, $X=$ $\left\{C_{i}\right\}_{i=1 \ldots n}$, of planar surfaces that satisfies the general position requirements. By the definition of $n(f, \phi)$, there exists a set, $\Delta=\left\{D_{i}\right\}_{i=1 \ldots n(f, \phi)}$, that can be used to construct a branched surface from $(f, \phi)$. Assume that $n \neq n(f, \phi)($ so $X \neq \Delta)$. In the proof of Proposition 3.1, we showed that $\Delta$ having cardinality $n(f, \phi)$ is sufficient to conclude that for every $D_{j} \in \Delta$, there exists an $x \in \cup_{i=1 \ldots n}\left\{C_{i}\right\}$ that flows under $\phi^{-1}$ into $D_{j}$ (before meeting the interior of some other element in $\Delta$ ). We then used this to show that $X$ may be altered by extending, contracting, or substituting its elements in $f$ until each $D_{j}^{+} \in \Delta^{+}$is surjectively covered by some element of $X$. After these modifications, some element of $X$, say $C_{0}$, may be omitted and we would still have a set that satisfies the first general position requirement for the construction of a branched surface from $(f, \phi)$ (since $n>n(f, \phi)$ ). Therefore, the orbit of any point in $C_{0}$ flows under $\phi^{-1}$ into one of the remaining elements of $X$. So a collection of connected subsets of $\cup_{i \neq 0}\left\{C_{i}\right\}$ flows under $\phi$ onto $C_{0}$. Further, only slight additional contractions or extensions of the remaining elements in $X$ are necessary to obtain a set that satisfies all the general position requirements. The cardinality of the complement for the new branched surface is $n-1$.

This procedure for reducing the number of components in $X$ (and hence in $M$ $N(V)$ ) may be repeated until we can no longer modify a collection of components in $M-N(V)$ with moves in $f$ so that a subset of their boundaries covers some $C_{j}^{+} \in X^{+}$that is not in the collection. In other words, we may continue to reduce the cardinality of by deleting elements of $X$ until we can no longer alter the remaining set so that it satisfies the general positions requirements.

\section{Appendix B.}

Condition $\mathrm{C}$ of the equivalence relation is difficult to check as stated. Here we find sufficient conditions for transitivity which, in some cases, is a straightforward exercise. Recall the proof of Theorem 4.2. If $W_{2}$ and $V_{2}$ can always be chosen so that substitutions in $f_{2}$ are not necessery to obtain conjugacy, then to ensure transitivity we need only check that changing critical connecting curves to strong connecting does not interfere with the structure preserving property of conjugacy maps. So we assume $W_{2}$ and $V_{2}$ can always be chosen in this manner.

We let $W_{1}$ be a branched surface constructed from $\left(f_{1}, \phi\right)$ using $\Delta_{1}=\left\{D_{i}^{1}\right\}_{i=1 . n}$ and we let $W_{2}$ be a branched surface constructed from $\left(f_{2}, \phi\right)$ using $\Delta_{2}=\left\{D_{i}^{2}\right\}_{i=1 \ldots n}$. Assume that $W_{1}$ and $W_{2}$ are $f_{1}-f_{2}$ counterparts. We denote the complement, $|j-2|+1$, of $j \in\{1,2\}$ by $j_{c}$. Let $B_{i}^{j}$ be the component of $M-N\left(W_{j}\right)$ that collapses to $D_{i}^{j}$. Suppose we may change a critically connecting curve of $W_{j}$ to strong connecting in $f_{j}$ by replacing $D_{i}^{j} \in \Delta_{j}$ with some surface $E_{i}^{j}$ that flows injectively 
onto $D_{i}^{j}$ under $\phi$ (or $\phi^{-1}$ ). As before, we may assume each such alternation changes the maximum number of critical connecting curves in $f_{j}$ with ends in $\pi_{W_{j}}\left(\partial B_{\imath}^{j}\right)$, to strong connecting. If each such alternation changes all critical connecting curves in $f_{j}$ with ends in $\pi_{W_{j}}\left(\partial B_{i}^{j}\right)$, then there are only a finite number of these substitutions that can be used to alter $\Delta_{j}$, at most one for each $i$.

We now find conditions under which this is the case. For each $i$ such that $\pi_{W_{3}}\left(\partial B_{i}^{j}\right)$ contains the ends of a critically connecting curve, we consider a fiber of $N\left(W_{j}\right)$ through $\partial B_{i}^{j}$. We take a closed, connected subset $I$ of this fiber such that all leaves through $I$ contain a surface that bijectively projects, along fibers, onto some hemisphere of $\partial B_{i}^{j}$. There is a set $A$ consisting of oriented immersions of $S^{1}$ in $W_{j}$, each contained in the union of $\pi_{W_{j}}\left(\partial B_{i}^{j}\right)$ with a critically connecting curve (in $f_{j}$ ). We require that each curve in $A$ begins at $\pi_{W_{j}}(I)$. For $\alpha \in A$, let $\operatorname{Inv}(\alpha)$ be the set of all points in $I$ which map back to $I$ along a holonomy map of $f_{j}^{*}$ (the corresponding foliation of $N\left(W_{j}\right)$ ) over $\alpha$. By the properties of $\alpha \in A, \operatorname{Inv}(\alpha)$ contains at least one fixed point under this map. Further, $\operatorname{Inv}(\alpha)$ is closed and connected. So if $\{\operatorname{Inv}(\alpha) \mid \alpha \in A\}$ satisfies the finite intersection property, then $\cap_{\alpha}\{\operatorname{Inv}(\alpha)\}$ is nonempty. We may then take the lowest (with respect to the orientation of $I$ ) point in $\cap_{\alpha}\{\operatorname{Inv}(\alpha)\}$. This point is fixed under the holonomy map of any curve in $A$. The surface $E_{i}^{j}$ will be in the leaf through this point since the substitution changes all critically connecting curves in $f_{j}$ with ends in $\pi_{W_{j}}\left(\partial B_{\imath}^{j}\right)$ to strong connecting.

Assume now that the above conditions are satisfied. If we can choose these successive substitutions in $\Delta_{j}$ and $\Delta_{j}$ so that branched surfaces constructed at each stage from the altered sets are $f_{1}-f_{2}$ counterparts, such substitutions do not interfere with the structure preserving property of the conjugacy map. So we focus on means to verify this condition is satisfied at each stage.

To avoid confusion with $W_{1}$ and $W_{2}$, we let $U_{j}$ (for $j \in\{1,2\}$ ) be the branched surfaces constructed from $\left(f_{j}, \phi\right)$ after we substitute $E_{i}^{j}$ for $D_{\imath}^{j}$ in $\Delta_{j}$ for some $i$. Since $W_{1}$ and $W_{2}$ are conjugate, there is a conjugacy map $h$ from $U_{1}$ onto $U_{2}$. We must find a way to verify that $h$ respects the $f_{1}-f_{2}$ structure.

Let $\gamma$ be a connecting curve of $W_{j}$ that is changed to a strong connecting curve in $f_{j}$ when we substitute $E_{i}^{j}$ for $D_{i}^{j}$ in $\Delta_{j}$. There is a closed integral curve of $f_{j}^{*}$ through $E_{i}^{j}$ that lies over $\gamma$ in $W_{j}$. (E.q. take any curve over $\gamma$ and through the integral strip of $f_{j}^{*}$ that becomes a strong connecting strip after the substitution. Join its ends with a curve in $E_{i}^{j}$ to obtain the desired closed curve.) It was shown in [8] that if $W_{1}$ and $W_{2}$ are $f_{1}-f_{2}$ counterparts, then for each set $C_{j}$ of closed curves in some leaf $L_{j}$ of $f_{j}^{*}$, there exists a set of closed curves $C_{j_{c}}$ in some leaf $L_{j_{c}}$ of $f_{j_{c}}^{*}$ such that $\pi_{W_{1}}\left(C_{1}\right)$ is mapped injectively onto $\pi_{W_{2}}\left(C_{2}\right)$ under the conjugacy map $g$ that respects the $f_{1}-f_{2}$ structure. In fact, $\pi_{W_{1}}\left(L_{1}\right)$ is mapped injectively onto $\pi_{W_{2}}\left(L_{2}\right)$. So once we have chosen $E_{i}^{j}$, we may always choose $E_{i}^{j_{c}}$ to ensure the image of the new $\gamma$ under $h$ (when $j=1$ ) or $h^{-1}$ (when $j=2$ ), is strong connectingin $f_{j_{c}}$. Since each alteration of $\Delta_{j}$ changes all critical connecting curves with ends in $\pi_{W_{j}}\left(\partial B_{i}^{j}\right)$ to strong connecting, then the same is true for alterations of $\Delta_{j_{c}}$.

Now for $j \in\{1,2\}$, consider the component of $\partial\left[M-N\left(U_{j}\right)\right]$ that collapses to $E_{\imath}^{j}$ and let $K_{j}$ be the set of connecting strips that have both ends in this component. 
Let $X_{j}$ be the corresponding set of connecting curves. We note that $h$ maps each element of $X_{1}$ injectively onto some element of $X_{2}$. Since the conjugacy map $g$ from $W_{1}$ onto $W_{2}$ respects $f_{1}-f_{2}$ structure, whether or not $h$ respects the $f_{1}-f_{2}$ structure depends entirely on the manner in which the connecting curves in $X_{1}$ and $X_{2}$ are crossed by $f_{1}$-curves and $f_{2}$-curves, respectively. In particular, if $E_{i}^{j}$ is contained in a compact leaf $L_{j}$ of $f_{j}$, then by [8] we may choose $E_{i}^{j_{c}}$ in a homeomorphic leaf $L_{j_{c}}$ of $f_{j_{c}}$ for which $g * \pi_{W_{1}}\left(L_{1}\right)=\pi_{W_{2}}\left(L_{2}\right)$. In this case, $U_{1}$ and $U_{2}$ are $f_{1}-f_{2}$ counterparts.

For the more general case, we consider integral curves $\alpha^{*}(t)(0 \leq t \leq 1)$ of $f_{j}^{*}$. Let $\alpha=\pi_{W_{j}}\left(\alpha^{*}\right)$. We require that some curve in $\alpha$ lies an element of $X_{j}$ and that $\alpha^{*}(0) \in E_{i}^{j}$. If $\alpha$ is closed, we take the integral curve $f_{\alpha}^{j}(t)(0 \leq t \leq 1)$ (of $f_{j}$ ) that $\alpha^{*}$ yields when we collapse the components of $M-N\left(W_{j}\right)$. If for each closed $\alpha$ (as above) in the branch set of $W_{1}, f_{\alpha}^{1}(1) \geq f_{\alpha}^{1}(0)$ as $f_{h(\alpha)}^{2}(1) \geq f_{h(\alpha)}^{2}(0)$ (where $\geq$ determined by relative position along $\Phi)$, then $h$ respects the $f_{1}-f_{2}$ structure.

\section{References}

[1] J. Christy and S. Goodman, Branched surfaces carrying codimension one foliations, Working paper.

[2] J. Christy, Branched surfaces and attractors I: Dynamic branched surfaces, Emory University 1990.

[3] W. Floyd and U. Oertel, Incompressible surfaces via branched surfaces, Topology, 23 (1984), 117-125.

[4] Morris W. Hirsch, Stability of compact leaves of foliations, Dynamical Systems, ed. by Peixoto, Academic Press (1973), 135-153.

[5] R.C. Penner, Combinatorics of train tracks, Annals of Mathematical Study, 125, Princeton University Press, Princeton New Jersey (1992).

[6] J. Plante, Anosov flows, American Journal of Mathematics, (1972), 729-754.

[7] G. Reeb, Sur certaines propriétés topologiques des variétées feuilletées, Actualités Sci. Indust, (1952) Hermann, Paris.

[8] S. Shields, Branched surfaces and the stability of compact leaves.

[9] The stability of foliations of orientable 3-manifolds covered by a product.

[10] W.P. Thurston, On geometry and dynamics of diffeomorphisms of surfaces, Bulletin of the American Mathematical Society, 19 (1988), 417-431.

[11] R.F. Williams, Expanding attractors, Institut des Hautes Etudes Scientifiques, Publications Mathematiques, 43 (1973), 473-487.

Received May 10, 1994.

College of Charleston

Charleston, SC 29424-0001

E-mail address: shields@math.cofc.edu 


\title{
PACIFIC JOURNAL OF MATHEMATICS
}

\author{
Founded in 1951 by
}

\author{
E. F. Beckenbach (1906-1982) $\quad$ F. Wolf (1904-1989)
}

\section{EDITORS}

Sun-Yung A. Chang (Managing Editor) Robert Finn University of California

Los Angeles, CA 90095-1555

pacific@math.ucla.edu

\section{F. Michael Christ}

University of California

Los Angeles, CA 90095-1555

christ@math.ucla.edu

Nicholas Ercolani

University of Arizona

Tucson, AZ 85721

ercolani@math.arizona.edu
Stanford University

Stanford, CA 94305

finn@gauss.stanford.edu

Steven Kerckhoff

Stanford University

Stanford, CA 94305

spk@gauss.stanford.edu

Martin Scharlemann

University of California

Santa Barbara, CA 93106

mgscharl@math.ucsb.edu

\section{Gang Tian}

Massachusettes Institute of Technology

Cambridge, MA 02139

tian@math.mit.edu

\section{V.S. Varadarajan}

University of California

Los Angeles, CA 90095-1555

vsv@math.ucla.edu

Dan Voiculescu

University of California

Berkeley, CA 94720

dvv@math.berkeley.edu

\section{SUPPORTING INSTITUTIONS}

CALIF. INST. OF TECHNOLOGY CHINESE UNIV. OF HONG KONG MACQUARIE UNIVERSITY NEW MEXICO STATE UNIV. OREGON STATE UNIV. PEKING UNIVERSITY RITSUMEIKAN UNIVERSITY STANFORD UNIVERSITY TOKYO INSTITUTE OF TECHNOLOGY UNIVERSIDAD DE LOS ANDES
UNIV. OF ARIZONA

UNIV. OF BRITISH COLUMBIA

UNIV. OF CALIF., BERKELEY

UNIV. OF CALIF., DAVIS

UNIV. OF CALIF,, IRVINE UNIV. OF CALIF., LOS ANGELES

UNIV. OF CALIF., RIVERSIDE UNIV. OF CALIF., SAN DIEGO UNIV. OF CALIF., SANTA BARBARA UNIV. OF CALIF., SANTA CRUZ
UNIV. OF HAWAII

UNIV. OF MELBOURNE

UNIV. OF MONTANA

UNIV. NACIONAL AUTONOMA DE MEXICO

UNIV. OF NEVADA, RENO

UNIV. OF OREGON

UNIV. OF SOUTHERN CALIFORNIA

UNIV OF UTAH

UNIV. OF WASHINGTON

WASHINGTON STATE UNIVERSITY

The supporting Institutions listed above contribute to the cost of publication of this Journal, but they are not owners or publishers and have no responsibility for its contents or policies.

Manuscripts must be prepared in accordance with the instructions provided on the inside back cover.

The table of contents and the abstracts of the papers in the current issue, as well as other information about the Pacific Journal of Mathematics, may be found on the Internet at http://www.math.uci.edu/pjm.html.

The Pacific Journal of Mathematics (ISSN 0030-8730) is published monthly except for July and August. Regular subscription rate: $\$ 245.00$ a year (10 issues). Special rate: $\$ 123.00$ a year to individual members of supporting institutions.

Subscriptions, back issues published within the last three years and changes of subscribers address should be sent to Pacific Journal of Mathematics, P.O. Box 4163, Berkeley, CA 94704-0163, U.S.A. Prior back issues are obtainable from Kraus Periodicals Co., Route 100, Millwood, NY 10546.

The Pacific Journal of Mathematics at the University of California, c/o Department of Mathematics, 981 Evans Hall, Berkeley, CA 94720 (ISSN 0030-8730) is published monthly except for July and August. Second-class postage paid at Berkeley, CA 94704, and additional mailing offices. POSTMASTER: send address changes to Pacific Journal of Mathematics, P.O. Box 6143, Berkeley, CA 94704-0163.

PUBLISHED BY PACIFIC JOURNAL OF MATHEMATICS at University of California, Berkeley, CA 94720, A NON-PROFIT CORPORATION

This publication was typeset using AMS-LATEX,

the American Mathematical Society's TEX macro system.

Copyright (c) 1995 by Pacific Journal of Mathematics 


\title{
PACIFIC JOURNAL OF MATHEMATICS
}

\author{
Volume $175 \quad$ No. $1 \quad$ September 1996
}

Homogeneous Ricci positive 5-manifolds

Dimitri Alekseevsky, Isabel Dotti de Miatello and CARlos J.

FERRARIS

On the structure of tensor products of $\ell_{p}$-spaces

ALVARO ARIAS and JEFFREY D. FARMER

The closed geodesic problem for compact Riemannian 2-orbifolds

JosEPH E. BORZELLINO and BENJAMIN G. LORICA

Small eigenvalue variation and real rank zero

Ola Bratteli and George A. Elliott

Global analytic hypoellipticity of $\square_{b}$ on circular domains

So-CHIN CHEN

Sharing values and a problem due to C. C. Yang

XIN-Hou HuA

Commutators and invariant domains for Schrödinger propagators

Min-JEI HUANG

Chaos of continuum-wise expansive homeomorphisms and dynamical properties of sensitive maps of graphs

\section{HiS AO KATO}

Some properties of Fano manifolds that are zeros of sections in homogeneous vector bundles over Grassmannians

\section{OLIVER KÜCHLE}

On polynomials orthogonal with respect to Sobolev inner product on the unit circle

XIN Li and FRANCISCO MARCELLAN

Maximal subfields of $\mathbf{Q}(i)$-division rings

STEVEN LIEDAHL

Virtual diagonals and $n$-amenability for Banach algebras

AlAN L. T. PATERSON

Rational Pontryagin classes, local representations, and $K^{G}$-theory

Claude Schochet

An equivalence relation for codimension one foliations of 3-manifolds

SANDRA SHIELDS

A construction of Lomonosov functions and applications to the invariant subspace problem

\section{ALEKSANDER SIMONIČ}

Complete intersection subvarieties of general hypersurfaces 